NIH, Lupus Foundation of America, Lupus Research Alliance, Merck Sharp and Dohme, NIAID, NIAMS, Pfizer, Rheumatology Research Foundation, Sanofi, and Takeda Pharmaceuticals) created to develop new ways of identifying and validating promising biological targets for diagnostics and drug development. Funding was provided through grants from the National Institutes of Health (UH2-AR067676, UH2-AR067677, UH2AR067679, UH2-AR067681, UH2-AR067685, UH2AR067688, UH2-AR067689, UH2-AR067690, UH2AR067691, UH2-AR067694, UM2-AR067678).

\section{LOW ATTENUATION NON-CALCIFIED CORONARY PLAQUES AND POSITIVE REMODELING INDEX: MARKERS OF VULNERABLE CORONARY PLAQUES IN SYSTEMIC LUPUS}

${ }^{1}$ George Stojan* ${ }^{2}$ Jessica Li, ${ }^{3}$ Laurence Magder, ${ }^{4}$ Matthew Budoff, ${ }^{2}$ Michelle Petri. ${ }^{1} J o h n s$ Hopkins University; ${ }^{2}$ Johns Hopkins University School of Medicine; ${ }^{3}$ University of Maryland; ${ }^{4}$ David Geffen School of Medicine at UCLA

\subsection{6/lupus-2019-Ism.274}

Background Positive remodeling index (RI) and presence of low attenuation noncalcified plaque (LANCP) are characteristic vessel changes in unstable coronary plaques. We sought to characterize LANCP and positive RI in patients with SLE.

Methods A total of 72 patients who met the ACR or SLICC classification criteria for SLE had CT angiogram studies, 30 of which had follow up CT angiograms. A total of 100 healthy controls who had two CT angiograms were included in the study. Each noncalcified plaque (NCP) detected within the vessel wall was evaluated for minimum CT density and vascular remodeling index. A LANCP was defined as an NCP with a density $<30$ Hounsfield units. Lesions with remodeling $0 \%$ were considered to have positive RI. T-test was used to evaluate baseline characteristics between lupus patients and controls. Paired t-test or Wilcoxon signed ranks test was used to compare LANCP volume and RI between baseline and follow-up. Fishers exact test was used to evaluate the association between change in LANCP, RI, demographic and clinical variables.

Results Lupus patients had a significantly higher burden of LANCP compared to healthy controls in all age subgroups except in those $>60$ years of age. LANCP volume was associated with age $(\mathrm{p}<0.01)$ and body mass index $(\mathrm{p}<0.01)$. No significant differences were observed between RI in lupus and controls at baseline. Despite a significant progression of the total noncalcified plaque burden in lupus compared to controls $(\mathrm{p}<0.0001)$, the LANCP in lupus patients regressed $(p<0.001)$. No demographic or clinical differences were observed between lupus patients whose LANCP progressed and those whose LANCP regressed. Lupus patients who were not treated with statins had a more significant regression of their LANCP burden $(\mathrm{p}<0.01)$ compared to controls who were on statins, while lupus patients who were taking statins had a significant progression $(\mathrm{p}<0.01)$.

Conclusions Lupus patients have a significantly higher burden of LANCP compared to healthy controls in all age subgroups except in those $>60$ years of age. The LANCP burden regresses more rapidly over time in lupus compared to controls. Surprisingly, the most significant LANCP plaque volume regression was seen in lupus patients who were never treated with statins, while the most significant progression was observed in those taking statins. Positive RI was ubiquitous, with no evidence of progression or differences compared to controls. These characteristic vessel changes may identify SLE patients at need for more frequent noninvasive cardiac monitoring.

Funding Source(s): The Hopkins Lupus Cohort was funded by AR 69572.

\section{GENOME-EXPOSOME INTERACTIONS IN SYSTEMIC LUPUS ERYTHEMATOSUS}

${ }^{1}$ Soren Jacobsen*, ${ }^{2}$ Theis Lange, ${ }^{3}$ Christoffer Collins, ${ }^{4}$ Constance Jensina Ulff-Møller, ${ }^{4}$ Henrik Christian Bidstrup Leffers. 'Lupus and Vasculitis Clinic, Rheumatology, Copenhagen University Hospital, Rigshospitalet; ${ }^{2}$ Center for Statistical Science, Peking University, Beijing, China; ${ }^{3}$ Department of Rheumatology, MedStar Washington Hospital Center, Washington DC, USA; ${ }^{4}$ Lupus and Vasculitis Clinic, Rheumatology, Copenhagen University Hospital, Rigshospitalet

\subsection{6/lupus-2019-Ism.275}

Background Systemic lupus erythematosus (SLE) is a systemic inflammatory autoimmune disease characterized by a broad spectrum of clinical and serological manifestations. This may reflect a complex and multifactorial etiology involving several identified genetic and environmental factors, though not explaining the full risk of SLE. Established SLE risk genotypes are either very rare or with modest effect sizes and twin studies indicate that other factors besides genetics must be operative in SLE etiology. The exposome comprises the cumulative environmental influences on an individual and associated biological responses through the lifespan. It has been demonstrated that exposure to silica, smoking and exogenous hormones candidate as environmental risk factors in SLE, while alcohol consumption seems to be protective.

Methods A systematic search on published studies that have investigated interactions between genetic and environmental risk factors resulted in five studies based on three different populations. Available measures of interaction were extracted.

Results Very few $(n=5)$ studies have investigated potential gene-environment interactions to determine if some of the unexplained SLE risk is attributable hereto. Even less $(n=2)$ have focused on interactions between specific risk genotypes and environmental exposures relevant to SLE pathogenesis. Cohort and case-control studies may provide data to suggest such biological interactions and various statistical measures of interaction can indicate the magnitude of such. However, such studies do often have very large sample-size requirements and it is suggested that the rarity of SLE to some extent can be compensated by increasing the ratio of controls.

Conclusions This review summarizes the current body of knowledge on gene-environment interactions in SLE. We argue for the prioritization of studies that comprise the increasing details available of the genome and exposome relevant to SLE as they have the potential to disclose new aspects of SLE pathogenesis including phenotype heterogeneity.

Funding Source(s): Gigtforeningen

\section{BASELINE RENAL AUTOPHAGY-RELATED PROTEIN P62 EXPRESSION LEVEL PREDICTS 24-WEEK TREATMENT EFFECTIVENESS IN LUPUS NEPHRITIS}

${ }^{1}$ Qiong Fu*, 'Shuhui Sun, ${ }^{1}$ Jie Chen, ${ }^{2}$ Chunde Bao. ${ }^{1}$ Shanghai Jiaotong University; ${ }^{2}$ Renji Hospital, School of Medicine, Shanghai Jiao Tong University, China

10.1136/lupus-2019-Ism.276 